\title{
Experimental Investigation of the Cavitation Effects on the Heat Generation in a Closed Loop Pumping System
}

\author{
${ }^{1}$ David Bermejo*; ${ }^{1}$ Xavier Escaler; ${ }^{2}$ Matevz Dular; ${ }^{1}$ Rafael Ruíz; \\ ${ }^{1}$ Universitat Politècnica de Catalunya BarcelonaTech, Barcelona, SPAIN; ${ }^{2}$ University of Ljubljana, Ljubljana, SLOVENIA
}

\begin{abstract}
A series of tests were carried out in the cavitation tunnel of the Laboratory for Water and Turbine Machines of the University of Ljubljana in order to investigate the effects of cavitation in the water heating process of closed loop pumping systems. For that, the water temperature was measured at the low pressure reservoir during runs of about 10 minutes. As expected, a constant temperature increase was observed which was proportional to the pump's rotation speed. Then, the results obtained while keeping the same pump operating point and flow conditions with and without cavitation in the Venturi test section were compared. Surprisingly, it was found that the temperature increase rate was slightly higher when cavitation is present in the system. Moreover, the heat power was always higher than the hydraulic one for all the tests up to a certain cavitation level. However, this trend was inversed for higher cavitation numbers and lower hydraulic powers. Both results seem to prove that there is a clear influence of cavitation in heat generation process. Therefore, a new test rig will be built in Barcelona by the Spanish company Condorchem Envitech, S. L. with the help of Universitat Politècnica de Catalunya BarcelonaTech to continue the current research.
\end{abstract}

Keywords: hydrodynamic cavitation; heat generation; temperature increase

\section{Introduction}

The cavitation process consists on the generation, growth and collapse of a cavity fulfilled with vapour inside a liquid due to a reduction in the local pressure. As pointed out by C. E. Brennen [1], cavitation differs from boiling by the mechanism which leads the mass transfer from liquid phase to gas phase: while in boiling the leading mechanism is the rising saturation pressure up to liquid's pressure by increasing the temperature, in cavitation the change on the state is led by the pressure decrease.

Since 1873 when O. Reynolds [2] presented the phenomenon of cavitation, the studies on cavitation have been disseminated in three main fields: bubble dynamics modelling, prediction of erosion in hydraulic machinery and search for positive effects in hydraulic systems.

The most extended approach for modelling cavity dynamics is the Rayleigh-Plesset equation, introduced by Lord Rayleigh in 1917 [3]. Actually, that field of research is full of analytical and computational studies about the morphology and dynamics of bubble clusters [1].

Historically, the effort has been focused on the way to avoid the negative effects of cavitation: erosion, noise, vibrations and the performance losses [1]. The work of R. T. Knapp [4] showed that erosion appears in the downstream stagnation point of the main cavity, where the bubbles collapse near the surface due to the raising pressure, instead of the point where the bubbles nucleate.

However, the studies about positive effects of cavitation have grown up during the last decades. For instance, J. Ozonek [5], P. R. Gogate [6] or A.G. Chakinala [7] have developed acoustic and hydrodynamic cavitation devices to be used as chemical reactors. Free hydroxyl radicals are produced during bubble collapse due to the high local temperatures and pressures, and they catalyse chemical reactions in the neighbourhood of the cavities. Moreover, such efforts are also employed for waste water treatment, as R. Cirimina [8] and M. Petkovsek [9] have pointed out.

Similar steps have been done in the food processing industry, as indicated by the works of M. Martynenko [10], who tested the performance of a hydrodynamic device as homogenizer, and the invention of C. Isopo [11], which is a rotary

*Corresponding Author, David Bermejo: david.bermejo@upc.edu 
device for heating and vaporizing beverages. Finally, the work of M. Petkovsek [9] also indicates that a modified centrifugal cavitation pump can enhance the disintegration of raw sludge in anaerobic digestion processes.

On the other hand, the thermodynamic or thermal effects of cavitation are well known because they control the growth and collapse of the cavity [1]. During the bubble growth, the mass transferred from liquid phase to vapour phase absorbs the liquid latent heat through the interface. As a consequence, the surrounding liquid is cooled and the saturation pressure decreases. This effect is higher in cryogenic liquids, as those used in rockets, than in water at room temperature [12]. Experimentally, the thermal effect has been visualized with a thermographic camera during the growing stage by M. Petkovsek [13].

However, there are other fields of interest related to thermodynamics and cavitation. On one hand, there are some studies, as those of B. Shneider [14] or J. Cai [15], which analyse the effects of cavitation on heat transfer through natural convection. In natural convective processes, the cavitation enhances heat transfer due the turbulence lead by the collapsing bubbles in the thermal boundary layer [14].

The prediction of very high pressures and temperatures at the end of the cavity collapse has inspired many inventions in terms of the so called hydrodynamic heat generators. Inventors and manufacturers of hydrodynamic heaters claim for the ability of cavitation to increase their efficiency. An example is the device patented by J. L. Griggs [16] which is a cylindrical drum with radial blind holes rotates at high speed surrounded by water.

Several authors have studied the performance and efficiency of such devices. The experiments carried out by K. B. Pyun [17] showed about $90 \%$ average thermal efficiency for different flow conditions. The work of S. M. Tahmid [18] described a hydrodynamic device that could rise the temperature of $113 \mathrm{l}$ of water from $30^{\circ}$ to $70^{\circ} \mathrm{C}$ in a closed circuit in less than half an hour with a $15 \mathrm{~kW}$ electric engine, while Mukesh [19] designed and tested a device with a $370 \mathrm{~W}$ electric engine that heated water from $25^{\circ}$ to $60^{\circ} \mathrm{C}$ with a thermal efficiency of $83 \%$ at least. However, most of the companies selling such devices claim for thermal efficiencies that appear to be unrealistic.

Regarding the heat generation process associated to cavitation, the fact is that water temperature in closed pumping systems, as in cavitation tunnels [20], is known to increase during operation and, because of that, they require a cooling system to control it. However, the mechanism by which the water raises its temperature is not fully understood.

To the author's knowledge there is no clear and reliable evidence in the literature of the cavitation contribution to the heating process. In this sense, the Universitat Politècnica de Catalunya BarcelonaTech (UPC) has started a research program in collaboration with the Spanish company Condorchem Envitech, S. L. to develop an optimized heat generator for wastewater treatment technologies in Zero Liquid Discharge facilities (ZLD). As a first step, the planned objective is to put light on the opened questions about the influence of cavitation on the heat generation in pumping systems.

For that propose, it is intended to build a test rig, based on the existing cavitation tunnel at the Laboratory for Water and Turbine Machines of the University of Ljubljana [13], in order to perform the experimental tests in Barcelona. This test trig consists of a closed loop water circuit driven by a variable speed centrifugal pump. The test section where cavitation can be generated and visualized will be located between two large reservoirs. A series of instruments measuring flow rate, pressure and temperature will permit to control and monitor the system operation conditions. Since the tunnel is not ready yet, the initial tests presented here were carried out at the cavitation tunnel of the University of Ljubljana.

\section{Description of the experiments and results}

The Ljubljana cavitation tunnel was operated at fixed operating conditions and delivered hydraulic power, thus keeping the flow rate and the pump head, with and without cavitation in the test section. Several runs of about $600 \mathrm{~s}$ duration each were carried out at different operating conditions. During the tests, the temperature of the circulating water was monitored with a probe located at the suction side reservoir. Moreover, the pump rotation speed, head and flow rate, and the absolute pressure at the inlet of the Venturi were also controlled. A high speed video camera was used to determine the cavity morphology, size and dynamic behaviour during the tests.

*Corresponding Author, David Bermejo: david.bermejo@upc.edu 
Table 1 shows a couple of testing conditions and the corresponding results, where $\bar{\sigma}$ is the average Sigma value as defined by (1) and computed as the average value between the cavitation number at the start and at the end of each trial, $t$ is the time, $\omega$ is the pump rotation frequency, $Q$ is the flow rate, $H$ is the pump head, $H_{\text {hyd }}$ is the hydraulic power as defined by (2), $P_{\text {in }}$ is the test section inlet pressure, $T_{\text {start }}$ and $T_{\text {end }}$ are the water temperatures at the start and at the end of each test, $T_{\text {slope }}$ is the temperature increase rate, $q$ is the heat power as defined by (3), $\Delta P$ is the pump pressure increase, $\rho$ is the water density, $g$ is the gravity, $\Delta h$ is the head difference between pressure measurements, $v_{2}$ and $v_{1}$ are the mean flow velocities at the pump discharge and intake, $m$ is the mass of water, $C_{P}$ is the water specific heat, $P_{\text {sat }}$ is the water vapour pressure and $v_{\text {throat }}$ is the mean flow velocity at the Venturi throat.

$$
\begin{gathered}
\sigma=\frac{P_{\text {in }}-P_{\text {sat }}(T)}{\frac{1}{2} \rho v_{\text {throat }}{ }^{2}} \\
N_{\text {hyd }}=Q\left[\Delta P+\rho g\left(\Delta h+\frac{{v_{2}}^{2}-v_{1}{ }^{2}}{2 g}\right)\right] \\
q=m C_{P} \frac{\Delta \mathrm{T}}{\Delta \mathrm{t}}
\end{gathered}
$$

As an example, the temperature evolution with cavitation on the Venturi for a pump rotation speed of $25 \mathrm{~Hz}$, a flow rate of $78 \mathrm{l} / \mathrm{min}$, a pump pressure increase of $93.2 \mathrm{kPa}$ and an inlet absolute pressure of $141.7 \mathrm{kPa}$ at the test section has been plotted in Figure 1. As it can be seen, the water temperature increases continuously at a constant rate indicating that heat power is being supplied to the liquid by the system that comprises the pump, the piping, the valves, the reservoirs and the Venturi test section. For this example, the calculated slope of the linear regression indicates that the temperature increase rate is of about $0.036^{\circ} \mathrm{C}$ per minute.

\begin{tabular}{|c|c|c|c|c|c|c|c|c|c|c|c|c|}
\hline Test & $\begin{array}{c}\text { Venturi } \\
\text { condition }\end{array}$ & $\begin{array}{c}\bar{\sigma} \\
{[-]}\end{array}$ & $\begin{array}{c}\boldsymbol{t} \\
{[\mathbf{s}]}\end{array}$ & $\begin{array}{c}\boldsymbol{\omega} \\
{[\mathbf{H z}]}\end{array}$ & $\begin{array}{c}\boldsymbol{Q} \\
{[\mathbf{l} / \mathbf{m i n}]}\end{array}$ & $\begin{array}{c}\boldsymbol{\Delta P} \\
{[\mathbf{k P a}]}\end{array}$ & $\begin{array}{c}\boldsymbol{N}_{\text {hyd }} \\
{[\mathbf{W}]}\end{array}$ & $\begin{array}{c}\boldsymbol{P}_{\text {in }} \\
{[\mathbf{k P a}]}\end{array}$ & $\begin{array}{c}\boldsymbol{T}_{\text {start }} \\
{\left[{ }^{\circ} \mathbf{C}\right]}\end{array}$ & $\begin{array}{c}\boldsymbol{T}_{\text {end }} \\
{\left[{ }^{\circ}{ }^{\circ} \mathbf{C}\right]}\end{array}$ & $\begin{array}{c}\boldsymbol{T}_{\text {slope }} \\
{\left[{ }^{\circ} \mathbf{C} / \mathbf{s}\right]}\end{array}$ & $\begin{array}{c}\boldsymbol{q} \\
{[\mathbf{W}]}\end{array}$ \\
\hline 1 & Cavitation & 1.64 & 655 & 25 & 78.0 & 93.2 & 125.0 & 141.7 & 28.717 & 29.142 & $6.05 \mathrm{E}-04$ & 151.8 \\
\hline 1 & No cavitation & 2.79 & 623 & 25 & 78.0 & 93.9 & 125.9 & 239.0 & 30.699 & 31.056 & $5.69 \mathrm{E}-04$ & 142.6 \\
\hline 2 & Cavitation & 1.63 & 250 & 35 & 126.0 & 181.0 & 387.8 & 362.0 & 28.104 & 28.625 & $2.00 \mathrm{E}-03$ & 500.9 \\
\hline 2 & No cavitation & 1.96 & 433 & 35 & 128.0 & 181.0 & 394.0 & 448.0 & 28.828 & 29.729 & $1.97 \mathrm{E}-03$ & 493.7 \\
\hline
\end{tabular}

Table 1. Measured and computed parameters for a couple of tests with and without cavitation while keeping the same pump performance. 


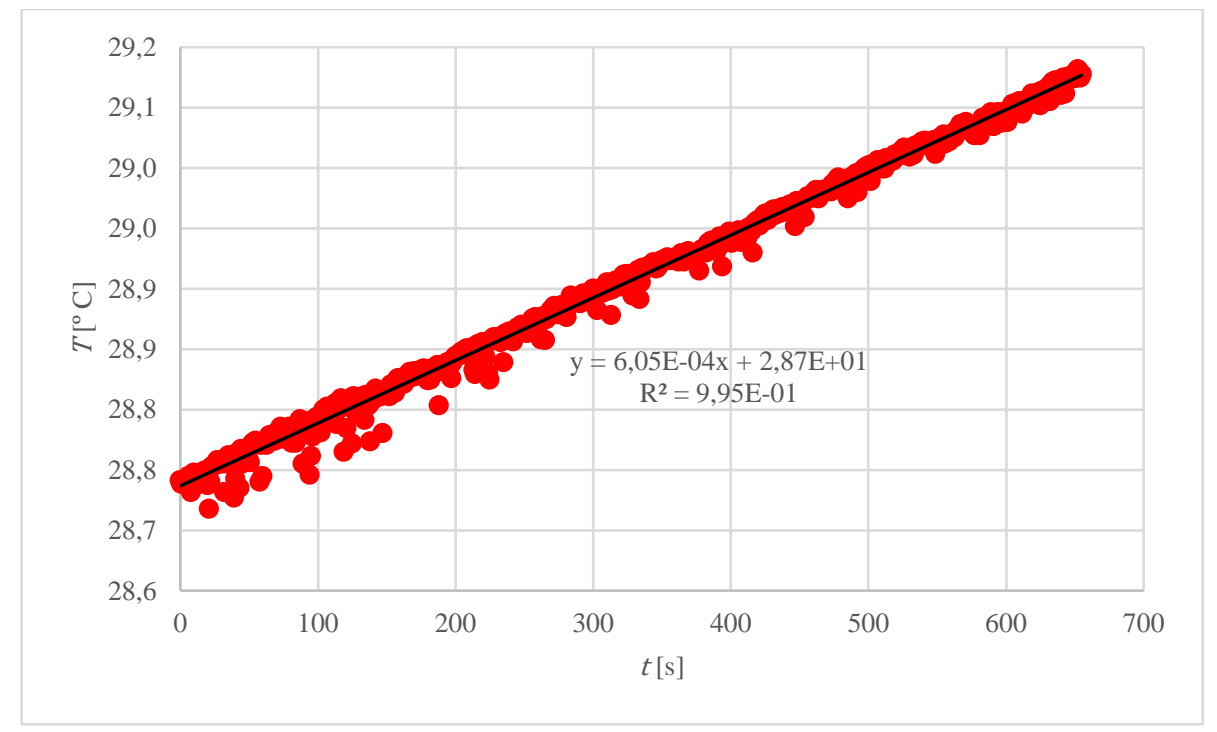

Figure 1. Water temperature inside the suction tank as a function of elapsed time with cavitation in the Venturi section, lineal trend line and equation.

\section{Discussion}

For each test, the slope of the linear regression obtained from curve fitting the temperature evolution in time was calculated. All the slope values have been plotted as function of the pump rotation speed in Figure 2. To enable a better understanding of the results, the data collected with cavitation in the test section has been represented with a red circle, meanwhile the data without cavitation has been represented with a blue triangle.

As expected, it has been confirmed that the temperature increase rate is proportional to the pump rotation speed, i.e. the energy delivered by the pump to the system. And surprisingly, the results also seem to indicate that the temperature increase rate is slightly higher when cavitation takes place in comparison with the same conditions without cavitation.

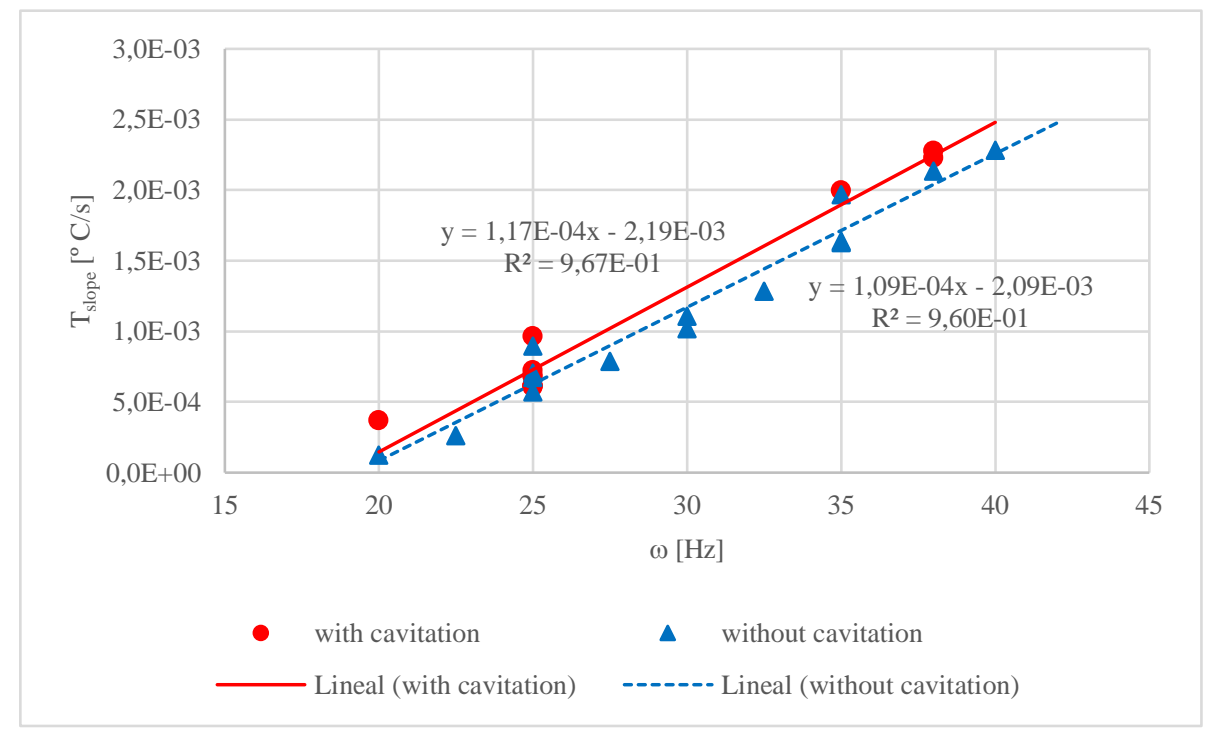

Figure 2. Temperature increase rate as a function of pump rotation frequency. Red circles indicate presence of cavitation while blue triangles indicate no cavitation in the system.

*Corresponding Author, David Bermejo: david.bermejo@upc.edu 
In Figure 3, the hydraulic power with cavitation (crosses) and without cavitation (pluses) and the heat power with cavitation (circles) and without cavitation (triangles) have been plotted as a function of the cavitation number. To help understanding, red colour represents again results with cavitation while blue colour represents those obtained without cavitation.

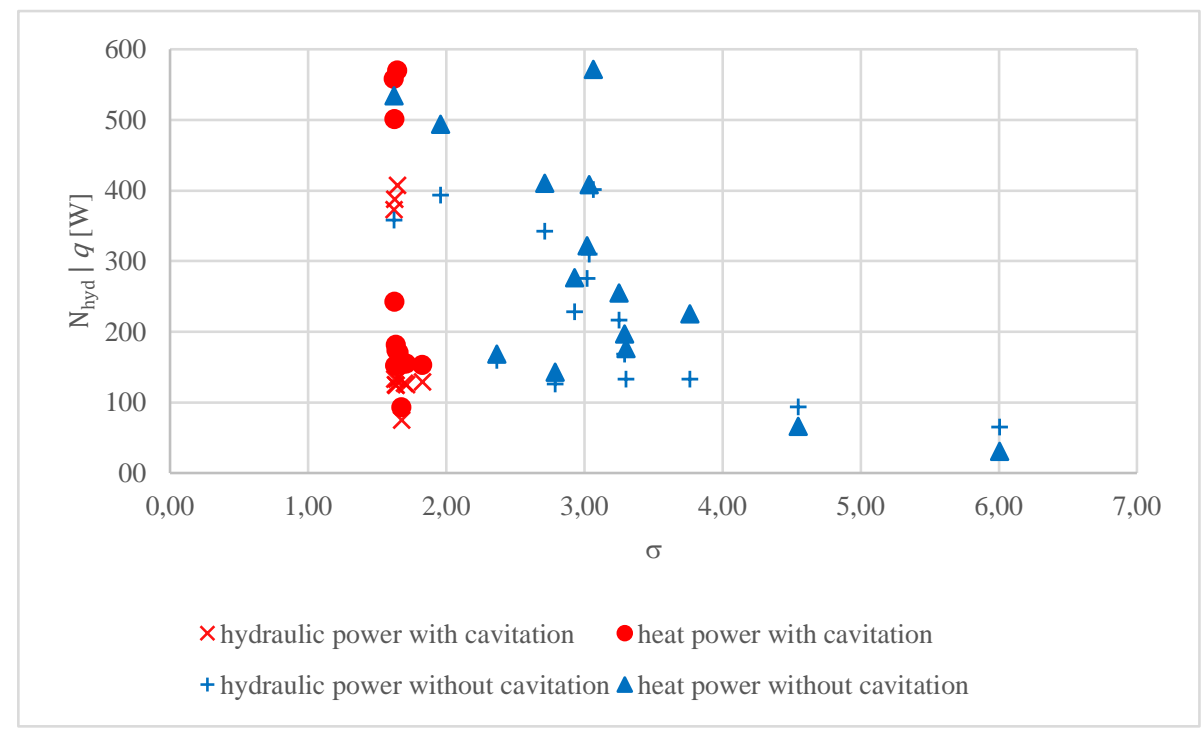

Figure .3 Hydraulic power with cavitation (crosses) and without cavitation (pluses), and heat power with cavitation (circles) and without cavitation (triangles) plotted as a function of the cavitation number. Red colour indicates cavitation while blue colour indicates no cavitation

It can be observed that the minimum cavitation number that could be achieved during the tests was around 1.62. This behaviour might be explained by a blockage of the test section. For those runs with cavitation in the Venturi, the cavitation number was always within the range from 1.62 to 1.83 . Only one test without cavitation fell inside that range. Another observation is that for cavitation numbers below 4 the heat power is higher than the hydraulic power. On the contrary, for the two tests at higher cavitation numbers the tendency switches and the heat power is lower than the hydraulic power. It must be noted that in particular for these two tests, the pump hydraulic power was the lowest among all the cases and below $100 \mathrm{~W}$.

\section{Conclusion}

A series of tests were carried out in the cavitation tunnel of the Laboratory for Water and Turbine Machines of the University of Ljubljana to investigate the possible relationship between flow thermodynamics and cavitation. For that, the same flow conditions were generated with and without cavitation in the test section for direct comparison. As a result, a slightly higher temperature increase rate was measured when cavitation was presented compared to the case without cavitation.

Another observation was that the heat power was higher than the hydraulic power for mean cavitation numbers below 4, but for higher values of sigma the tendency switched and the hydraulic power was higher than the heat power. It must be noted that in these cases the pump worked far from its best efficiency point.

The observed differences without and with cavitation seem to prove that cavitation has an influence in the heating generation process in closed loop pumping systems, but it does not explain the high thermal efficiency claimed by inventors and manufacturers of hydrodynamic heaters.

These experiments have provided a very valuable knowledge for designing and operating a new test rig that will be used to develop an optimized heat generator for wastewater treatment technologies in Zero Liquid Discharge facilities (ZLD). This device will be constructed in Barcelona by the Spanish company Condorchem Envitech, S. L. with the supervision of Universitat Politècnica de Catalunya BarcelonaTech. 


\section{References}

[1] Brennen, C. E. (1995). Cavitation and bubble dynamics. Oxford University Press

[2] Reynolds, O. (1873). The causes of the racing of the engines of screw steamers investigated theoretically and by experiment. Transactions of the Royal Institution of Naval Architects. 14 (56-67).

[3] Lord Rayleigh, O. M. (1917). VIII. On the pressure developed in a liquid during the collapse of a spherical cavity. The London, Edinburgh and Dublin Philosophical Magazine and Journal of Science. 34 (200).

[4] Knapp, R. T. (1955). Recent investigations of the mechanics of cavitation and cavitation damage. Transactions of ASME. 77 (1045).

[5] Ozonek, J., Lenik, K. (2011). Effect of Different Design Features of the Reactor on Hydrodynamic Cavitation process. Archives of Materials Science and Engineering. 52 (2).

[6] Gogate, P. R., Pandit, A. B. (2000). Engineering design methods for cavitation reactors II: hydrodynamic cavitation. American Institute of Chemical Engineers Journal 46 (8).

[7] Chakinala, A. G., Gogate, P. R., Burgess, A. E., Bremner, D. H. (2006). Intensification of hydroxyl radical production in sonochemical reactors. Ultrasonics Sonochemistry 14 (509).

[8] Cirimina, R., Albanese, L., Meneguzzo, F., Pagliaro, M. (2016). Wastewater remediation via controlled hydrocavitation. Environmental Reviews 25 (2).

[9] Petkovsek, M., Mlakar, M., Levstek, M., Strazar, M., Sirok, B., Dular, M. (2015). A novel rotation generator of hydrodynamic cavitation for waste-activated sludge disintegration. Ultrasonics Sonochemistry 26 (408).

[10] Martynenko, A., Astatkie, T., Satanina, V. (2015). Novel hydrodynamic food processing technology. Journal of Food Engineering. 152 (8).

[11] Isopo, C. (2010). Centrifugal rotary device for heating and/or vaporizing liquids. United States Patent. US 7647896 B2.

[12] Yoshida, Y., Sasao, Y., Okita, K., Hasegawa, S., Shimagaki, M., Nakamura, N. (2006). Influence of thermodynamic effect on synchronous rotating cavitation. Sixth International Symposium on Cavitation CAV2006.

[13] Petkovsek, M., Dular, M. (2015). Experimental study on the thermodynamic effect in a cavitating flow on a simple Venturi geometry. $9^{\text {th }}$ international Symposium on Cavitation CAV2015.

[14] Cai, J., Huai, X., Liang, S., Li, X. (2010). Augmentation of natural convective heat transfer by acoustic cavitation. Frontiers of Energy and Power Engineering in China. 4(3).

[15] Schneider, B., Kosar, A., Kuo, C. J., Mishra, C., Cole, G. S., Scaringe, R. P., Peles, Y. (2006). Cavitation enhanced heat transfer in microchannel. Journal of Heat Transfer. 128 (1293).

[16] Griggs, J. L. (1993). Apparatus for heating fluid. United States Patents. 5188090.

[17] Pyun, K. B., Kwon, W. C., Oh, K. T., Yoon, J. Y., (2011). Investigation of the performance for heat generator using hydrodynamic cavitation. Proceedings of the ASME-JSME-KSME AJK-Fluids2011.

[18] Tahmid, S. M., Gill, B. S., Park, K. M., Ryu, Y., Lee, S. H. (2013). Optimization and testing of a first generation cavitation heat pump. $120^{\text {th }}$ ASEE Annual Conference \& Exposition.

[19] Mukesh, Shetty, N. P. (2015). Design and fabrication of externally driven liquid cavitation heating system. International Research Journal of Engineering and Technology. 02 (03).

[20] Staff of Lewis research Center (1963). Facilities and techniques employed at Lewis research center in experimental investigations of cavitation in pumps. Symposium on Cavitation Research Facilities and Techniques.

*Corresponding Author, David Bermejo: david.bermejo@upc.edu 